\title{
ENHANCE KNOWLEDGE COMMUNICATION AND LEARNING: A SURPRISE PARADOX
}

Ruth Chatelain-Jardon ${ }^{1}$, https://orcid.org/0000-0001-9079-2678

Jesus S Carmona ${ }^{1}$, https://orcid.org/0000-0001-6757-1367

Jose Luis Daniel ${ }^{1}$, https://orcid.org/0000-0002-5228-854X

${ }^{1}$ Texas A\&M University, Kingsville, USA

\begin{abstract}
Human-computer interface is a pivotal factor that can promote or deter the effectiveness of Web-based knowledge communication. There is abundant research that strain to improve interfaces by considering user needs through usability studies; however, few researches consider the incorporation of automatic brain mechanisms in order to improve knowledge communication performance. The objective of this research is not to establish a relationship between the negative stimulus presence and improved knowledge communication, but rather to show that the shape of this function follows the Yerkes-Dodson Law. Partial least squares (PLS) was utilized to analyze the data. Results found in this study support the evidence that surprising negative events enhance knowledge communication effectiveness, but more importantly that the surprise-performance relationship is not a linear function but follows the inverted U shape.
\end{abstract}

Keywords: Human Computer Interaction, Web Interface Design, Automatic Brain Mechanisms, Enhanced Memorization Capacity, Web-based Learning

Manuscript first received: 2018-09-28. Manuscript accepted: 2018-11-14

Address for correspondence:

Ruth Chatelain-Jardon. Texas A\&M University, Kingsville, USA

E-mail: ruth.chatelain@tamuk.edu

Jesus S Carmona. Texas A\&M University, Kingsville, USA

E-mail: jesus.carmona@tamuk.edu

Jose Luis Daniel. Texas A\&M University, Kingsville, USA

E-mail: jose.damiel@tamuk.edu 


\section{INTRODUCTION}

Knowledge communication is one of the activities that has been drastically affected by the development and popularization of the Internet, the World Wide Web (www) and mobile devices. Together, these technologies form a medium that through the use of a variety of resources brings unlimited potential to support the creation of different types of educational experiences for teaching and learning (Schnitman, 2007).

It is important to note that a pivotal factor that would promote or deter the effectiveness of the Web-based knowledge communication is the human-computer interface since it is the point of contact between the human and the computer, and it is the one in charge of the communication between them. Abundant research has been accumulated in studies that try to improve interfaces by considering user needs through usability studies Schnitman (2007); but, few studies have considered the incorporation of automatic brain mechanisms in the design of human-computer interfaces (see Kock et al., 2008, 2008a, 2009 \& Chatelain et al., 2016), in order to improve knowledge communication performance. These few studies have proposed the selective incorporation of simulated web-based negative stimulus (regardless of the nature of the threat) into the computer interface in order to enhance knowledge communication effectiveness/memorization throughout the activation of the automated mechanism of surprise. Even though, the studies above mentioned successfully prove that the incorporation of web-simulated threats improve knowledge communication effectiveness; the objective of this research is not to establish a relationship between these two variables (negative stimulus presence, improved knowledge communication) but to show that the shape of this function/relationship follows an inverted U-curve. Thus, the present study aims to demonstrate that the strength of the negative stimulus presented in the human computer interface directly impacts the level of enhanced knowledge communication effectiveness shown by the subjects.

\section{LITERATURE REVIEW}

Increased availability of cheaper and more effective and efficient computers has sturdily influenced the proliferating use of information and communication technology (ICT). Ubiquitous technology, aided by the popularization of the Internet, the World Wide Web (Web) and mobile devices, has resulted in the creation of new forms of communication. Knowledge communication is one of the activities that has been drastically affected by these developments since they bring unlimited potential to support the creation of different types of educational experiences for teaching and learning (Schnitman, 2007). Regardless of its common questioning about its effectiveness in relation to individual student needs and perceptions; web-based knowledge communication provides a flexible, cost-effective alternative to traditional learning environments (Picciano, 2002). Millions of students participate in online, hybrid, mixed-mode, and Web-enhanced face-to-face courses; in fact, the Babson Survey Research Group (2017) reported that in the United States, during 2016, 6,359,121 students (31.6\% of all students) were enrolled in at least one online course. However, online learning is not exclusive for schools, Universities and students; virtually all the organizations have implemented online training in at least one of their many areas. In fact, the 2017 Training Industry Report (2017) states that in the United States at least $84 \%$ of the organizations was providing mandatory or compliance training mostly online. Given the importance of ICT in its role as an organizational learning enabler Real et al, (2006), it is of crucial significance to enhance the knowledge communication effectiveness/ memorization created/communicated throughout the human computer interface. Previous studies 
by Kock et al, (2008, 2008a, 2009) and Chatelain et al, (2016) have successfully proven that the incorporation of web-simulated threats enhance knowledge communication effectiveness; however, they fail to recognize that the strength of the negative stimulus presented in the human computer interface directly impacts the level of enhanced knowledge communication effectiveness shown by the subjects.

\section{Psychology, Brain Mechanisms and the Surprise Model}

Study of the human side of the human-computer interaction (HCI) is imperative given the ubiquity of computers and devices throughout our day-to-day life. Psychology becomes a vital player in the development of human-computer interfaces because the interaction with computers is a human activity. Psychology is so important in the HCI field that even the name of the field (including the word "human") leads to the establishment of an apparently strong role of psychology within the discipline (Newell \& Card, 1985). In like manner, brain mechanisms seem to be rooted at the core of psychology; as Buss (1995) mentions "all psychological theories imply evolved mechanisms" (p. 1) and even though, why or how these brain mechanisms work goes beyond the realm of this research; what is important to emphasize is that "once those mechanisms are in place, they can be activated or executed in ways that may ... lead to fitness in current environments" (Buss, 1995, p.10). The studies by Kock et al, (2008, 2008a, 2009) and Chatelain et al, (2016) have used evolutionaryand technology-related web-simulated negative stimulus (threats) to activate the automatic brain mechanisms of surprise. Please note that the surprise model explains how this mechanism has been frequently linked to enhanced memorization capacity.

\section{The Yerkes-Dodson Law}

It was in 1908 when Robert M. Yerkes and John D. Dodson developed the Yerkes-Dodson inverted U-shaped performance law (Yerkes \& Dodson, 1908). The original version of his theory states that there is an optimal amount of arousal to achieve increased performance. When a subject is not aroused or over-aroused, the performance achieved by this subject is poor, when arousal is increased to a moderated (optimal) level, then the performance achieved is improved. The function that describes this arousal-performance relationship follows an inverted U-shaped curve. Basically, the idea behind this theory is that arousal/stimulation is productive until it reaches the optimal level, when the stimulations crosses the threshold of optimality, then the increasing stimulation will deter the performance. Given the versatility of this function, it has been applied to other similar relationships such as: anxiety-performance, stress-performance, dissatisfaction-performance, among many others (Johnston et al., 2012). It is even used to represent the capita national income-inequality in income distribution relationship (Kuznets' Inverted U-hypothesis). More in sync with the present study, Sotgiu and Galati (2007) found that the relationship between memory and stress follows a curvilinear shape, consistent with the Yerkes-Dodson law. 


\section{RESEARCH METHODOLOGY}

\section{Objective}

Previous studies have successfully proven that the incorporation of web-simulated threats improve knowledge communication effectiveness; however, the objective of this research is not to establish a relationship between these two variables (negative stimulus presence, improved knowledge communication) but to show that the shape of this function/relationship follows an inverted U-curve. Thus, the main objective of the present study is to attempt to demonstrate that the strength of the negative stimulus presented in the human computer interface directly impacts the level of enhanced knowledge communication effectiveness shown by the subjects.

\section{Hypotheses}

Surprising negative stimulus seems to enhance knowledge communication effectiveness about information that is acquired immediately after the surprising event; however, it would not be expected that all the subjects would be surprised/scared at the same level. As well, the level of performance presented by the subjects would not be expected to be the same for everyone. Thus, following the Yerkes-Dodson law, it is expected that subjects who reported to be slighter surprised by the stimulus would perform poorly compared to the subjects who expressed to be surprised; on the same path, it would be expected to have a poor performance if the subject reported to be extremely surprised. This discussion would lead to the following two hypotheses:

H1: Knowledge communication effectiveness when the surprise is mild will be lower than when the surprise is moderate.

H2: Knowledge communication effectiveness when the surprise is extreme will be lower than when the surprise is moderate.

Please note that this is hypothesized based on the belief that even though it has been demonstrated that the automatic mechanism of surprise gets stronger stimulation (and hence, greater level of memory enhancement) by a surprising negative stimulus (or threats) compare to neutral or positive surprises; it would be expected that a surprise extremely negative (a deadly threat) would defeat the purpose of this study, since the subjects would "freeze" and their cognitive effort will be focus on other aspects of survival rather than memorization (to know more about the fight, fly or freeze response to surprise, please refer to Schmidt et al., 2008).

\section{Model}

The graphical representation of the hypotheses is presented in figure 1 .

Please note that several control variables have been added to the model in order to try to exclude other potential effects that are unrelated to the strength of the surprising negative stimulus to

which the subjects have been exposed. The potential effects of the control variables over knowledge communication effectiveness are represented as dotted arrows since their existence and sign are uncertain at this time. 


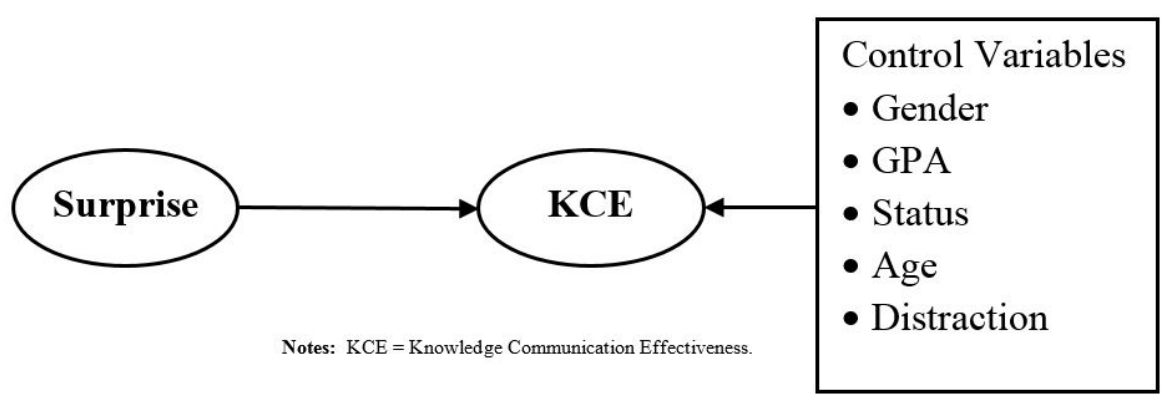

Figure 1. Graphical Representation of the Model

\section{Methodology}

Considering that the main objective of the present study is to attempt to demonstrate that the strength of the negative stimulus presented in the human computer interface directly impacts the level of enhanced knowledge communication effectiveness shown by the subjects, experimentation is needed. A web-based learning experiment was conducted so that performance-related data from subjects exposed to a surprising web-simulated negative stimulus (threat) would self-report their level of surprise. This level of surprise will be linked to their performance and this relationship is expected to follow the Yerkes-Dodson function.

As part of the experiment, the subjects were asked to review six web-based learning modules, after reviewing half of the modules, they were exposed to a surprising web-simulated negative stimulus (computer fatal error message screen) and then, they completed the review of the remaining three learning modules.

The experiment was time-controlled, thus all the subjects reviewed not only each module during the exact same time interval, but also they were exposed to the surprising negative stimulus at exactly the same time. With purposes of evaluating the knowledge communication effectiveness for each module, at the end of the last module reviewed, the subjects were asked to take an online test covering the content just reviewed. The test had three multiple-choice questions per module, each question had four possible answers of which only one was correct, and no pre-defined answer was selected in any of the questions asked. Additionally, they were asked to complete an online questionnaire in which they answered questions about the level of surprise generated by the surprising web-simulated negative stimulus (threat), demographics, prior knowledge about the content and stimulus-related general scariness level.

\section{Instrument}

The instrument here utilized was adopted from the research by (Kock et al., 2008). The reported levels of validity and reliability of the instrument were adequate.

The independent variable for this study would not be the presence per se of the surprising web-simulated negative stimulus in the Web-based knowledge communication task; but the strength or level of surprised generated by the surprising threat. This strength will be measured in a 7-point Likert scale, which is self-reported by the subjects exposed to the surprising stimulus. 
The expected consequence of the presence/strength of the surprising web-based negative stimulus (independent variable) is the enhanced knowledge communication effectiveness in an inverted-U shape. In other words, it is expected that the subjects will improve their performance as the strength of the stimulus increases until it reaches the "optimal" moderate level, after this threshold has been surpassed the performance would start decreasing as the strength of the stimulus continues increasing. Therefore, the inverted- $U$ shaped enhanced knowledge communication effectiveness is the dependent variable.

In order to avoid potential effects that are not related to this relationship, several variables of control were included. The control variables included in this study are: age, gender, GPA, scholastic status, years (demographical) and stimulus-related distraction (perceptual).

\section{Sample}

The sample size for this experiment was 240; the subjects were between 16- and 49-years old with an average age of 24.65 years. Almost half of the subjects (49\%) were males and the average working experience of the sample was 4.66 years with a range from 0 to 28 years. Please note that none of the subjects was familiar with the content of the experiment prior to their participation in it.

\section{Research Method}

Variance-based structural equation modeling with the partial least squares (PLS) method (Chin, 1998); Gefen, Straub and Boudreau (2000) was utilized to analyze the data. The reflective measurement model was verified for validity and reliability throughout the implementation of factor analysis, coefficients of reliability, Cronbach's Alpha coefficients Hair, Anderson and Tatham, (1987), and the average variance extracted (AVE) for each latent variable (Fornell \& Larcker, 1981).

\section{Validity and Reliability}

A test for convergent validity was conducted in order to make sure that all the indicators belong to their respective constructs. The recommended threshold for the all items is a value of .5 or above (Hair et al., 1998). Table 1 presents the results the factor analysis, and all the indicators presented a value higher than .5. In addition, the model was analyzed for discriminant validity in order make sure that all variables differ from each other (Hair et al., 1998). One way to test for discriminant validity is by analyzing the average variance extracted (AVE) numbers. The test involves that the AVE value of each construct should be greater than the correlations associated with that construct. Table 2 presents the results of the AVE, and the values in parentheses present values higher than the other correlations. With respect to reliability, the model was assessed with Cronbach's Alpha. It has been noted that acceptable values to be higher than 0.7. For this research all Cronbach's alpha values are higher than 0.7 .

The last analysis conducted was a full collinearity approach. This approach measures for vertical and lateral collinearity. In addition, it has been noted to be a technique for also measuring common method biased (Kock and Lynn, 2012). The proposed threshold for each latent variable is a value of 3.3 or less. The results of the full collinearity tests are presented in Table 3. All the values present a value lower than 3.3, therefore we can conclude that multicollinearity and common method biased is not an issue for this research 
Next, the results of the validity and reliability tests for the reflective measurement model used for the perceptual control variables are presented.

Table 1. Factor loadings Surprise and Knowledge Communication Effectiveness

\begin{tabular}{lc}
\hline Surprise & $(\mathbf{0 . 9 7 2}) *$ \\
\hline I was scared by the stimuli & 0.911 \\
The stimuli that appeared on the screen made me scared & 0.968 \\
The stimuli made me scared & 0.972 \\
I felt fear when I saw the stimuli on the screen & 0.952 \\
\hline Knowledge communication Effectiveness & $(\mathbf{1 . 0 0 0}) *$
\end{tabular}

* Cronbach's Alpha is presented in parenthesis

Table 2. Correlation Among Variables

\begin{tabular}{|c|c|c|c|c|c|c|c|}
\hline & SURP & $\mathrm{KCE}$ & DIST & GENDER & GPA & STATUS & AGE \\
\hline 1. SURP & $(0.951)$ & & & & & & \\
\hline 2.KCE & -0.032 & $(1.000)$ & & & & & \\
\hline 3.DIST & $0.441 * * *$ & 0.021 & (0.898) & & & & \\
\hline 4.GENDER & -0.077 & -0.053 & $-0.025 * *$ & $(1.000)$ & & & \\
\hline 5.GPA & $0.202 *$ & 0.099 & 0.056 & 0.064 & $(1.000)$ & & \\
\hline 6.STATUS & 0.110 & -0.039 & -0.040 & 0.015 & $0.212 * *$ & $(1.000)$ & \\
\hline 7.AGE & 0.077 & 0.004 & -0.063 & 0.011 & $0.259 * * *$ & 0.051 & $(1.000)$ \\
\hline
\end{tabular}

$\mathrm{SURP}=$ Surprise; $\mathrm{KCE}=$ Knowledge communication effectiveness;

Average Variance Extracted (AVE) are reported in parentheses.

Notes: $* * * * *, *$ indicate significant level at $.001,0.01$ and 0.05 respectively

Table 3. Full Collinearity Values

\begin{tabular}{ccccccc}
\hline SURP & KCE & DIST & GENDER & GPA & STATUS & AGE \\
1.318 & 1.022 & 1.316 & 1.054 & 1.179 & 1.065 & 1.085 \\
\hline
\end{tabular}

\section{RESULTS}

The statistically significant links show the corresponding $\beta$ coefficients near the arrows. Those $\beta$ coefficients suggest that the corresponding effects are unlikely to be due to luck since they were stronger than chance. Either an asterisk $\left(^{*}\right)$ or a double asterisk $(* *)$ follow each of the significant $\beta$ coefficients. The single asterisk $(*)$ indicates significance at the .05 level, and the double asterisk $(* *)$ refers to an effect at a significance level of .01 .

Figure 2 presents a diagram showing the results of the structural modeling analysis intended to test the relationship between web-simulated surprising negative stimulus and knowledge communication effectiveness, the results of the variables of control are also shown. 


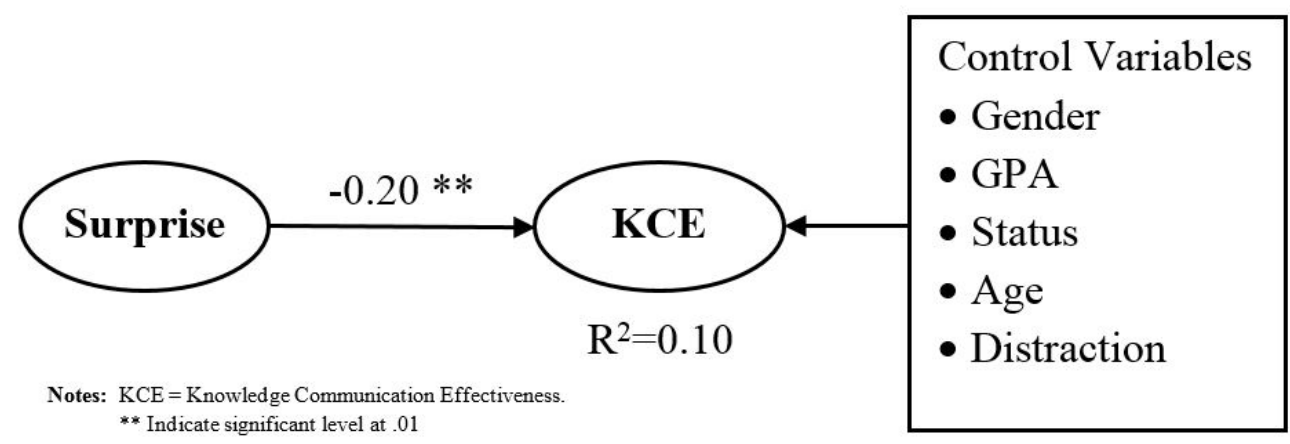

Figure 2. Statistical Results

From the demographic and perceptual control variables, two demographical variables significantly affected the surprising negative stimulus-knowledge communication effectiveness relationship. Those were GPA $(\beta=.22, \mathrm{P}<.01)$ and scholastic status $(\beta=.14, \mathrm{P}<.05)$.

Hypothesis 1 suggests that knowledge communication effectiveness when the surprise is mild will be lower than when the surprise is moderate and hypothesis 2 suggests that knowledge communication effectiveness when the surprise is extreme will be lower than when the surprise is moderate. When both hypotheses are put together, and inversed-U shaped relationship between the strength of the surprising negative stimulus and performance level is hypothesized. Figure 3 shows the standardized values of the relationship between the strength of the surprising negative stimulus and knowledge communication effectiveness. The first part of the graph fully supports hypothesis 1 , meanwhile the second part of the graph partially supports hypothesis 2 ; the latter is only partially supported given the fact that the curve does not decrease completely forming a perfect inverted-U.

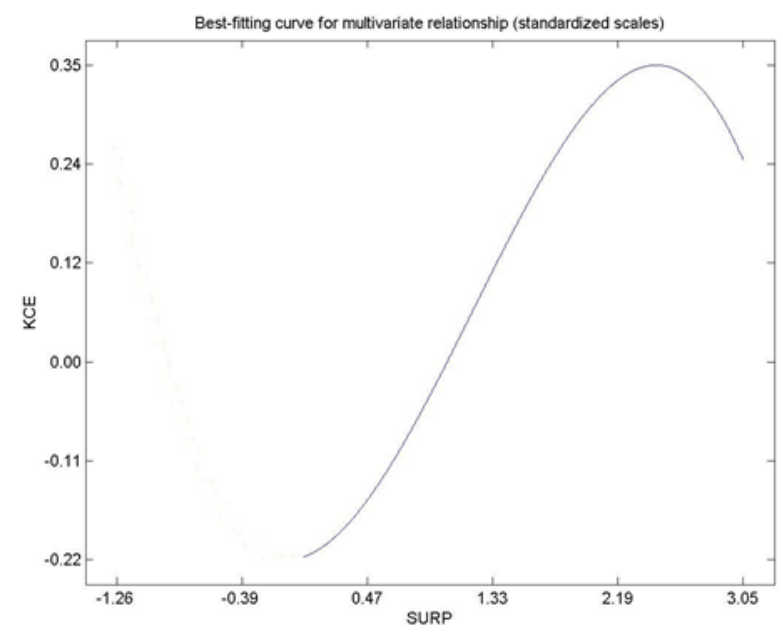

Figure 3. Best-fitting Curve (Standardized)

Starting from the baseline of zero standard deviations (mean), the function shown on Figure 3 supports the hypotheses posted in this study. Performance (knowledge communication effectiveness) steadily increases as the strength of the surprising negative stimulus goes from zero to 2.3 standard deviations. On the same path, knowledge communication effectiveness steadily decreases as the strength of the surprising negative stimulus keeps growing from 2.3 to 3.04 standard deviations. 
Figure 4 shows the inverted-U shaped relationship between the strength of the surprising negative stimulus and knowledge communication effectiveness, the scale values are shown without being standardized.

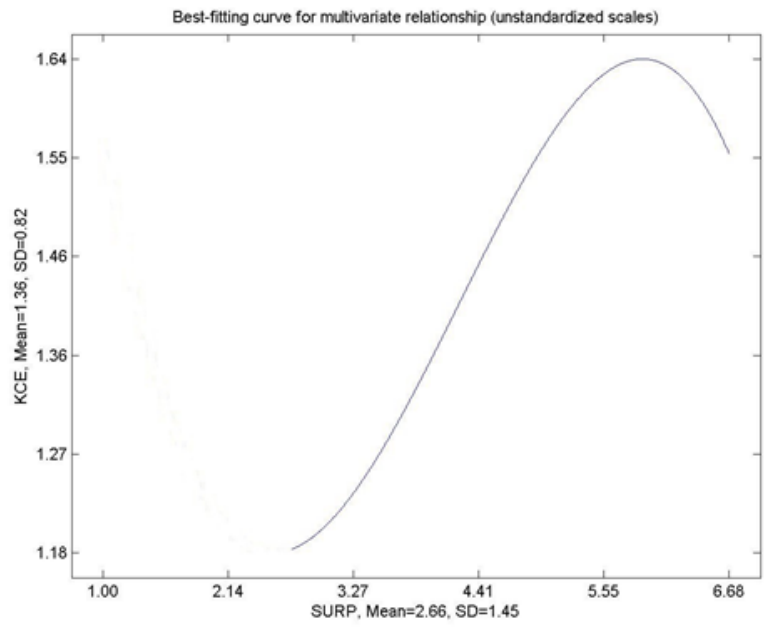

Figure 4. Best-fitting Curve (unstandardized)

The figure shown above suggests that, at least for this study, the optimal level of stimulation is 6 (6.04) in a 1-to-7 point scale. However, it is important to note that the effects of the surprising negative stimulus start showing their influence over knowledge communication effectiveness (performance) at 2.66 stimulation level. Thus, as long as the web-simulated surprising negative stimulus had an effect above average (2.66) all the way up to 6, the subjects had an increasing performance; once the 6-point threshold was overpassed, the performance steadily drops.

\section{RESULTS AND DISCUSSION}

Both of the hypotheses posed by this study were supported by the data analysis results; this is summarized in Table 4.

Table 4. Summary of Hypotheses-Testing Results

\begin{tabular}{ll}
\hline Hypothesis & Outcome \\
\hline H1: Knowledge communication effectiveness when the & Fully supported \\
surprise is mild will be lower than when the surprise is & \\
moderate. & Partially supported \\
H2: Knowledge communication effectiveness when the & \\
surprise is extreme will be lower than when the surprise & \\
is moderate. &
\end{tabular}

It is important to remember that the objective of this study was to show that the relationship that exists between the strength of the surprising web-simulated negative stimulus and performance (knowledge communication effectiveness) follows an inverted-U shape as suggested by the YerkesDodson law. This curvilinear function aligns with the mechanism of surprise model since it has been 
proposed as a "threat detection system" which was designed to prioritize the processing of threatening stimuli (Schützwohl \& Borgstedt, 2005). And it is the prioritization of information processing resources the one that apparently leads to improved focusing of attention Meyer, Reisenzein and Schutzwohl, (1997) and, as a consequence, the enhancement of knowledge communication effectiveness (performance). As part of the results of this study, performance gets improved significantly by the presence of a surprising web-based simulated negative stimulus; however, this improvement keeps constant until it reaches the threshold of optimality on the strength of the surprising stimulus. In other words, the subjects exposed to the surprising web-simulated negative stimulus, improved their performance as the strength of the stimulus increased until it reached the "optimal" moderate level, after this threshold got surpassed, the performance started decreasing as the strength of the stimulus continued increasing. The optimal level of stimulation (threshold) suggested in this study is 6 in a 1-to-7 point scale, since the effects of the surprising negative stimulus started showing their influence over knowledge communication effectiveness (performance) at 2.66 stimulation level.

Regarding the control variables (demographical and perceptual), only two demographic variables shown a significant effect over knowledge communication effectiveness: GPA and scholastic status. This positive and significant connection between the demographical variable of control GPA and performance (knowledge communication effectiveness) has been documented in previous studies (Alhajraf \& Alasfour, 2014; Du and Wu, 2014; \& Masky, 2014). This literature reports an enduring relationship between GPA and performance, being the control variable a good predictor for the dependent variable. Scholastic status also showed a positive and significant effect over knowledge communication effectiveness; this link can be interpreted as a learning enrichment through the increased years of working experience. In other words, the more years of experience, better the performance. Additionally, Guile and Griffiths (2001) suggest that the more experience a person has, the more willingness for better performance exists.

\section{CONCLUSION}

In sum, this study was intended to demonstrate that the strength of a surprising web-simulated negative stimulus will influence in an inverted-U shape the performance (knowledge communication effectiveness) level achieved by the subjects exposed to the stimulus. The results found in this study support the understanding that surprising negative events enhance knowledge communication effectiveness, but even more importantly may be that this study shows that the surprise-performance relationship is not a linear function. Specifically, as long as the strength of the surprising stimulus increased until it reached the "optimal" moderate level, the performance shown by the subjects exposed to stimulation, improved. However, that performance started decreasing as soon as that threshold was surpassed by the strength of the stimulus. The optimal level of stimulation (threshold) suggested in this study is 6 in a 1-to-7 point scale, since the effects of the surprising negative stimulus started showing their influence over knowledge communication effectiveness (performance) at 2.66 stimulation level. In standardized terms, the optimal level of stimulation (threshold) suggested would be 2.3 standard deviations to the right of the mean, considering that the effects of the surprising negative stimulus would started showing their influence over knowledge communication effectiveness (performance) at the baseline of 0 standard deviations (mean). In other words, the effects of the surprising websimulated negative stimulus over knowledge communication effectiveness would start working from the average surprising effect that any unexpected stimulus may have on the subjects. 
This study provides robust support for further research efforts connecting surprising events to their behavioral effects particularly for the improvement of web-based knowledge communication effectiveness. In order to gain increased credibility, future research must be conducted to address potential limitations, mainly in research dealing with uncommon topics like the one revealed here.

\section{REFERENCES}

Alhajraf, N. M., \& Alasfour, A. M. (2014). The impact of demographic and academic characteristics on academic performance. International Business Research, 7(4), 92-100.

Babson Survey Research Group, (2016): Grade increase: Tracking distance education in the United States, Retrieved September, $4^{\text {th }} 2017$, from https://www.onlinelearningsurvey.com/highered.html

Buss, D.M. (1995). Evolutionary psychology: A new paradigm for psychological science. Psychological Inquiry, 6(1), 1-30.

Chatelain-Jardon, R., Carmona, J., \& Kock, N. (2016). An extension to simulated web-based threats and their impact on knowledge communication effectiveness. International Journal of Technology and Human Interaction, 12(3), 64-77

Chin, W.W. (1998). Issues and opinion on structural equation modeling. MIS Quarterly, 22(1), 7-16.

Du, C., \& Wu, J. (2014). The effect of human interactions on student performance and satisfaction of blended learning. Academy of Educational Leadership Journal, 18(3), 11-21.

Fornell, C., \& Larcker, D.F. (1981). Evaluating structural equation models with unobservable variables and measurement error. Journal of Marketing Research, 18(1), 39-50.

Gefen, D., Straub, D. W., \& Boudreau, M.-C. (2000). Structural equation modeling and regression: Guidelines for research practice. Communications of the AIS, 4(7), 1-76.

Guile, D. \& Griffiths, T. (2001). Learning through work experience. Journal of Education and Work, 14(1), 113-131.

Hair, J.F., Anderson, R.E., \& Tatham, R.L. (1987). Multivariate data analysis, $2^{\text {nd }}$ Edition. New York, NY: Macmillan.

Johnston CA, Moreno JP, Regas K, Tyler C, Foreyt JP. The application of the Yerkes-Dodson law in a childhood weight management program: examining weight dissatisfaction. J Pedi- atr Psychol. 2012;37:674-9.

Kock, N. (2018). WarpPLS User Manual: Version 6.0. Laredo, TX: ScriptWarp Systems

Kock, N., Chatelain-Jardon, R. \& Carmona, J. (2008). An experimental study of simulated web-based threats and their impact on knowledge communication effectiveness. IEEE Transactions on Professional Communication, 51(2), 183-197.

Kock, N., Chatelain-Jardon, R. \& Carmona, J. (2008) a. Incorporating simulated animal attacks in humantechnology interaction interfaces: The predictive power of biosemiotics and evolutionary psychology. International Journal of Technology and Human Interaction, 4(4), 68-87.

Kock, N., Chatelain-Jardón, R. \& Carmona, J. (2009), Scaring them into learning!? Using a snake screen to enhance the knowledge transfer effectiveness of a web interface. Decision Sciences Journal of Innovative Education, 7(2), 359-375.

Kock, N., \& Lynn, G.S. (2012). Lateral collinearity and misleading results in variance-based SEM: An illustrationand recommendations. Journal of the Association for Information Systems, 13(7), 546-580. 
Maksy, M. (2014). Factors associated with student performance in intermediate accounting: A comparative study at commuter and residential schools. The Journal of Applied Business and Economics, 16(5), 86108.

Meyer, W., Reisenzein, R., \& Schutzwohl, A. (1997). Toward a process analysis of emotions: The case of surprise. Motivation and Emotion, 21(3), 251-274.

Newell, A., \& Card, S.K. (1985). The prospects for psychological science in human-computer interaction. Human Computer Interaction, 1(3), 209-242.

Picciano, A.G. (2002). Beyond student perceptions: Issues of interaction, presence, and performance in an online course. Journal for Asynchronous Learning Networks 6(1), 21-40.

Real, J.C., Leal, A. \& Roldan, J.L. (2006). Information technology as a determinant of organizational learning and technological distinctive competencies. Industrial Marketing Management, 35(4), 505-521.

Schnitman, I. (2007). The Dynamics Involved in Web-based Learning Environment (WLE) Interface Design and Human-Computer Interactions (HCI): Connections with Learning Performance. Morgantown, West Virginia: West Virginia University.

Schmidt, N., Richey, A., Zvolensky, M., \& Maner, J. (2008). Exploring human freeze responses to a threat stressor. Journal of Behavior Therapy and Experimental Psychiatry, 39(3), 292-304.

Schützwohl, A. \& Borgstedt, K. (2005). The processing of affectively valenced stimuli: The role of surprise. Cognition \& Emotion, 19(4), 583-600.

Sotgiu, I. \& Galati, D. (2007). Long-term memory for traumatic events: Experiences and emotional reactions during the 2000 flood in Italy. The Journal of Psychology, 141, 91-108.

Yerkes, R. M., \& Dodson, J. D. (1908). The relation of strength of stimulus to rapidity of habitformation. Journalof Comparative Neurology, 18, 459-482. 